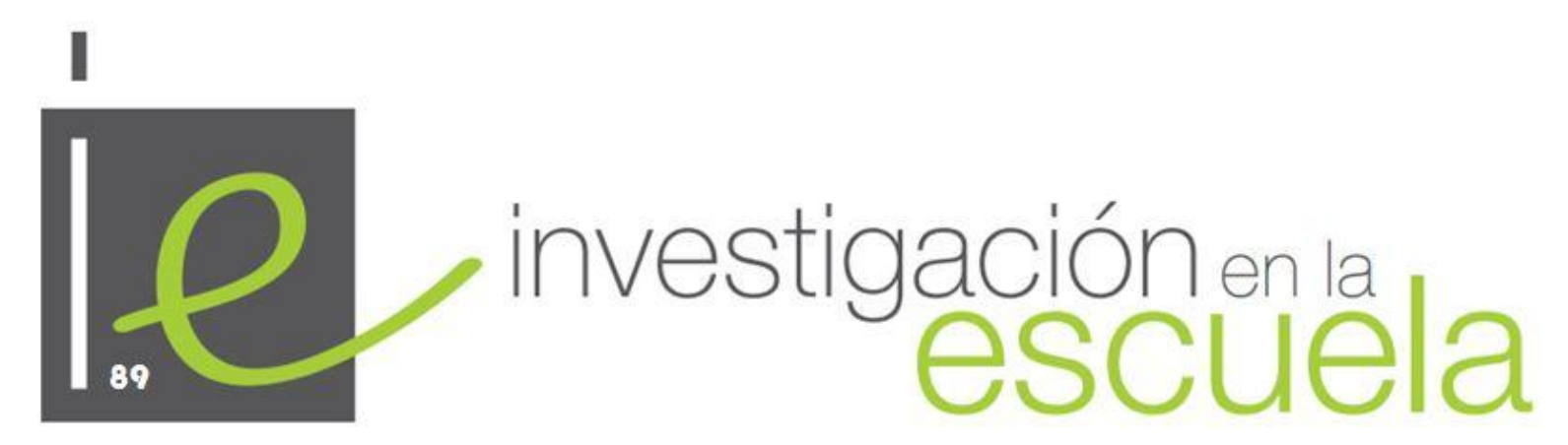

Revista académica evaluada por pares y de acceso abierto

\title{
Expresión corporal y educación emocional en alumnos de $3^{\circ}$ de educación primaria
}

\author{
Laura Luesia Labor. \\ María Rosario Romero Martín \\ Universidad de Zaragoza \\ España
}

Citación: Luesia, L. y Romero, M.R. (2016). Expresión corporal y educación emocional en alumnos de $3^{\circ}$ de educación primaria. Investigación en la escuela, 89, 49-70. Recuperado de:

http://www.investigacionenlaescuela.es/articulos/R89/R89-4.pdf

Resumen: El presente artículo responde a un trabajo de innovación educativa que pretende indagar en el mundo emocional del alumno de primaria desde la perspectiva de la Educación Física, concretamente desde la Expresión Corporal. Este estudio se realizó durante el curso académico 2009-2010, con el objeto de profundizar en la realidad emocional de un grupo de niños de tercero de educación primaria al experimentar un programa de Expresión Corporal centrado en el desarrollo de dos competencias emocionales, conciencia emocional y regulación emocional y, en consecuencia, conocer la influencia del programa en dichas competencias.

Palabras clave: "Expresión Corporal"; "educación Emocional"; "conciencia emocional"; "regulación emocional"; "educación primaria"; "educación física".

Body language and emotional education in 3th grade primary school students Abstract: The present article is the fruit of a educational innovation work that pretends to investigate the emotional world of the primary student, from the point of view of physical education, more particularly body language. This research was developed during the academic year 
2009-2010, with the aim of studying the emotional reality of a 3th grade primary school student group on experiencing a Body Language program, based on two basic emotional dimensions, emotional awareness and emotional regulation, so that consequently being able to achieve the influence of that program on that two basic emotional dimensions.

Key words: "Body Language”; “emotional education"; “emotional awareness”; "emotional regulation"; "primary school”; "physical education".

\section{Le langage du corps et l'éducation affective dans élèves de 3ème année d' éducation primaire}

Résumé: Cet article répond à un travail de innovation pédagogique qui pursuit ênqueter dans le monde affective de l'élève de primaire du point de vue de la perspective de la Éducation Physique, spécifiquement, dés le Langage du Corps. Cet étude a été réalisé pendant le course 2009-2010, avec l'objetif de réchercher dans la réalité affective d'un grupo d'enfants de 3ème année d'éducation primaire, à partir de l'experimentation d'un programme de Langage du Corps, axé sur le développement de deux compétences émotionnelles, la conscience du corps et la régulation émotionnelle, et, par conséquent, connaittre l'influence du programme dans cettes compétences. Mosts clé: "Langage du corps"; "éducation affective"; "conscience émotionnelle"; "régulation émotionnelle"; "éducation primaire"; "éducación physique”.

\section{Introducción}

La educación obligatoria pretende el desarrollo integral y global de la persona, incidiendo en las distintas dimensiones de la misma, entre las que se encuentra la dimensión afectivo-emocional. Sin embargo el análisis del desarrollo de los currículos muestra que su puesta en práctica se centra fundamentalmente en aprendizajes de carácter conceptual por encima de los vivenciales, quedando el desarrollo de aspectos emocionales relegados a un segundo plano (Álvarez, 2001). La evidencia de esta carencia, potenciada por el éxito de la obra de Goleman (1996) sobre la inteligencia emocional, ha hecho que en los últimos años haya tomado fuerza socialmente la necesidad de una educación emocional, la cual entendemos como el "proceso educativo, continuo y permanente que pretende potenciar el desarrollo emocional como complemento indispensable de desarrollo cognitivo, constituyendo ambos elementos esenciales del desarrollo de la personalidad integral" (Bisquerra, 2000: 243). No obstante, la realidad es que existe una falta de profesionales de este ámbito formados en educación emocional (Caballero, 2004) y también de planes formativos al respecto (Abarca, Barco y Sala, 2002).

La Educación Física (EF), por su propia idiosincrasia, fomenta ciertas competencias emocionales que quizás desde otras materias son más difíciles de desarrollar. La motricidad tiene la ventaja de suscitar en el alumnado emociones, sensaciones, pensamientos, ideas, estados de ánimo...(Gelpi, Romero-Martin, Rovira y Lavega, 2014; Lavega, Filella, Lagardera, Mateu y Ochoa, 2013; Torrents y Mateu, 2015), mientras se están desarrollando capacidades perceptivas, físicas o sociales. Recogiendo el argumento de Castañer y Camerino (2004: 221), "la condición humana representada por lo cognoscitivo, lo físico-motriz y lo socio-afectivo, siguen siendo atomizadas y por tanto, poco válidas si no apelan al enclave de intersección representado por lo afectivo y lo volitivo", nos atrevemos a señalar que desde la motricidad se puede enfatizar la dimensión afectivo-emocional del alumnado. De las diferentes manifestaciones de la motricidad, la Expresión Corporal (EC), disciplina cuya lógica interna se centra en la motricidad expresiva, comunicativa y estética (Romero Martín, 2015), es una de las que puede establecer un vínculo más directo con la emoción, ya que su eje vertebral se fundamenta sobre la dimensión socio-afectiva de la persona, “...es un modo de sentir interno que se 
da en el plano inconsciente y emocional de la persona en relación con todo aquello que le acontece" (Castañer y Camerino, 2004: 228), siendo por tanto, un vehículo adecuado, a priori, para el desarrollo de competencias emocionales.

El hecho de "...expresar y comunicar las emociones, ideas, pensamientos, sensaciones, vivencias..." tal y como argumenta Sierra (2001:33), nos lleva a pensar que el trabajo de lo emocional en EC es algo inherente a la propia disciplina, siendo su objetivo primordial conseguir el desarrollo emocional y perceptivo de la persona (Santiago, 1985). La EC se caracteriza por huir de las técnicas estereotipadas como patrones de la motricidad, porque la finalidad está en su propia práctica, porque lo importante es el proceso y no el resultado, por el tratamiento divergente en las respuestas de los alumnos y, sobre todo, porque la afectividad es el eje tanto de contenido como de método (Romero Martín, 2008). Esta autora propone el sentimiento y la emoción no solo como un objetivo, sino como un bloque de contenido en su propuesta de definición del campo propio de la EC, soporte en el que nos apoyaremos para tratar las emociones desde esta disciplina.

Como decíamos, el estudio que presentamos pretende, a través de la EF, indagar en la dimensión afectivo-emocional del alumno. Esta dimensión emocional queda recogida en cinco competencias emocionales, según la propuesta de Bisquerra (2003): conciencia emocional, regulación emocional, autonomía personal (autogestión), inteligencia interpersonal y habilidades de la vida y el bienestar. En nuestro caso, al tratarse de un estudio que debía ceñirse a un curso escolar, decidimos centrarnos en la base del proceso emocional y por tanto referirnos exclusivamente a dos competencias, conciencia emocional y regulación emocional. La conciencia emocional, pretende conocer las propias emociones y las emociones de los demás a través del análisis del propio comportamiento y el de los otros. Supone reconocer y utilizar el lenguaje de las emociones tanto en comunicación verbal como no verbal y comprender las causas y consecuencias de las mismas. Es la competencia fundamental del proceso emocional (Renom, 2003). La EC da pie a este proceso, a reconocer las emociones en uno mismo y en los demás, a comprenderlas e identificarlas, ya que una persona cuando expresa está plasmando sus sentimientos, emociones o visión de la vida, mediante sus movimientos, expresiones corporales y faciales, mediante la relación con los otros, o a través de sus manifestaciones fisiológicas (rubor, sudoración...), como se indica en Günter (1995), Castañer y Grasso (2006), Albaladejo (2007) o Ruano (2003). Por otro lado, la regulación emocional pretende enseñar a controlar aquellas emociones que afectan negativamente a uno mismo (Renom, 2003). La EC puede ayudar a ese control dirigiendo a los alumnos a la reflexión y autocontrol de las mismas; además a través de la motricidad se pueden llevar a cabo diferentes estrategias de regulación emocional para conseguir estos objetivos, como la relajación, el control consciente de la respiración, el contacto corporal, la liberación de energía mediante distintas acciones motrices o la imaginación creativa, estrategias de regulación de las emociones que en definitiva tratan de canalizar las emociones desagradables, tolerar la frustración y saber esperar y gestionar las gratificaciones.

Concluyendo, es importante apreciar que las emociones se relacionan con la expresión motriz y con la comunicación no verbal y verbal, pero teniendo presente la corporalidad en todas sus dimensiones y manifestaciones. Como indican Castañer y Camerino (2004: 219):

Sería una aberración pretender acotar las emociones a un cuerpo de conocimientos cerrado y objetivo... debemos contemplarlas como un sistema dinámico de vivencias singulares y objetivas que están siempre presentes en la vida de la persona y que evolucionan y se modifican a lo largo de la vida. 


\section{Diseño metodológico}

\section{Objetivo y contexto de la experiencia}

El objetivo que nos planteamos fue analizar las implicaciones de una unidad didáctica de EC en la vivencia emocional de alumnos de $3^{\circ}$ de primaria, concretamente en las competencias emocionales de conciencia y regulación emocional. Para ello se diseñaron varios instrumentos ad hoc que nos permitiesen el análisis del comportamiento emocional de los participantes. Esta unidad se realizó en el colegio Jesús María El Salvador de Zaragoza durante los meses de abril y mayo del curso 2009-2010 en horario de educación física (EF), martes (15:30 a 17:00) y jueves (11:45 a 12:30), y en momentos puntuales dentro del aula ordinaria.

\section{Participantes}

Después de pedir autorización y colaboración del centro, se analizaron los grupos a los que se tenía acceso, eligiendo al grupo de $3^{\circ} \mathrm{A}$, alumnos de 8 y 9 años, nacidos en el año 2001 por el equilibrio entre niños y niñas de esa clase (22 alumnos, 11 niños -50\%- y 11 niñas -50\%-).

\section{Elaboración de la Unidad didáctica}

La unidad didáctica formó parte de la programación anual de EF, aunque con un talante transversal ya que se incorporaron instrumentos en el aula ordinaria que requirieron la colaboración de la maestra tutora de los niños.

La UD constó de doce sesiones. Los objetivos fueron dos: (1) Tomar conciencia de las propias emociones y las emociones de los demás a través de la Expresión y Comunicación verbal y no verbal, identificando dichas emociones (alegría, tristeza, enfado, sorpresa, miedo, amor, vergüenza, nerviosismo) y (2) Conocer y saber aplicar en distintas situaciones estrategias motrices (relajación, respiración, liberación de energía, imaginación creativa) y no motrices para autorregularse ante situaciones de enfado, nerviosismo y tristeza, básicamente. Para el diseño se partió de la propuesta de Bisquerra (2000), a partir de Fernández-Abascal (1997), que tras la revisión de múltiples autores organiza las emociones en positivas (alegría, humor, amor y felicidad), en negativas (ira, miedo, ansiedad, tristeza, vergüenza y rechazo) y en ambiguas (sorpresa, esperanza y compasión). Teniendo en cuenta el nivel educativo de los alumnos, su desarrollo emocional, las fuentes bibliográficas sobre trabajo de las emociones en educación primaria y la discusión entre docentes, se seleccionaron para el desarrollo de la UD como emociones positivas: alegría y amor, como negativas: tristeza, ira (enfado), ansiedad (nerviosismo), miedo y vergüenza y como ambiguas: sorpresa.

Se decidió que el contenido de conciencia emocional predominara sobre el de regulación emocional en las dos primeras sesiones, ya que creíamos necesario conocer las emociones, vivenciarlas y experimentarlas, para posteriormente utilizar las estrategias de regulación motriz más adecuadas. En las últimas sesiones de la UD cobró más protagonismo el contenido de regulación emocional por los motivos mencionados. No obstante, la vuelta a la calma de todas las sesiones se planteó con una actividad de regulación emocional para que los alumnos volvieran a un estado de calma. La última parte de la sesión se dedicó a la reflexión escrita y personal de los alumnos ante distintas situaciones planteadas por la profesora. El alumno compartía libremente con el grupo la reflexión realizada. Posteriormente, la maestra recogía esas reflexiones para luego ser tratadas. Por otro lado, además de los recursos propios de EC y los estímulos sonoros, visuales y luminosos para suscitar determinadas emociones, se creó un rincón de recursos dentro del aula ordinaria para 
conseguir también allí el segundo objetivo que nos planteábamos en la unidad didáctica. En este rincón estaban ubicados "el bote vitamínico" y "las manos abiertas", dos estrategias planteadas para la regulación emocional del alumno dentro de la clase. El "bote vitamínico" era un bote transparente en el que cada alumno podía escribir un mensaje de apoyo, de alegría, de ánimo, de cosas bonitas... La idea era alegrar a otro compañero cuando se encontrase triste o bajo de ánimos. Los mensajes eran como las vitaminas a tomar. Cada alumno podía dejar o coger un mensaje cuando lo considerase. En cuanto a "las manos abiertas", se trataba de una manualidad de manos abiertas colocada en la pared que invitaba a dejar los malos pensamientos, enfados, nervios, etc., si durante diez segundos se presionaban con las propias manos. Las dos estrategias podían ser utilizadas libremente por los alumnos cuando ellos las necesitaran.

Por último, para desarrollar los objetivos, se elaboró un cuestionario que se pasó a los alumnos antes y después de la UD para observar la evolución, un diario que fue construyéndose durante la intervención y un registro de la utilización de estrategias de regulación emocional del aula ordinaria

\section{Procedimiento de la experiencia}

Para el desarrollo de esta experiencia se han seguido los siguientes pasos:

1. Elaboración de la unidad didáctica de EC y diseño del cuestionario, del diario, del registro del aula y protocolo de aplicación de todos ellos.

2. Aplicación del cuestionario previamente a la propuesta de enseñanza.

3. Implementación de la enseñanza, del diario y de la utilización de las estrategias en el aula habitual.

4. Aplicación del mismo cuestionario tras la conclusión de la unidad didáctica.

5. Análisis de los resultados y evaluación de la experiencia.

\section{Instrumentos de toma de información}

Cuestionario de emociones. En la revisión de antecedentes a este estudio no encontramos cuestionarios adaptados a nuestro campo de acción, la motricidad, más concretamente a la EC. Todos los cuestionarios (CEE, CEE-R, TMMS, TMMS-24) tenían un marcado carácter psicológico y se alejaban de nuestros objetivos y de nuestro prisma de aproximación al mismo. Por ello elaboramos uno basándonos en uno de los enfoques que Extremera y Fernández-Berrocal (2003) proponían para evaluar la inteligencia emocional a través de medidas de habilidad o de ejecución, compuesto por tareas emocionales que el alumno debía resolver. Estos autores se refieren al test MSCEIT (Test de Inteligencia Emocional Mayer-Salovey-Caruso) como medida de habilidad para evaluar la inteligencia emocional. El MSCEIT es un test de inteligencia emocional entendida como una aptitud. Esto quiere decir que algunas respuestas son mejores que otras y, por tanto, que algunas personas pueden mostrar más aptitud que otras en las tareas del test.

Así, en función de los objetivos de la unidad didáctica, extrajimos las siguientes variables para la elaboración del cuestionario:

1) Sociotipo: edad, curso, sexo.

2) Variable conciencia emocional de alegría, enfado, miedo, amor, tristeza, sorpresa, vergüenza y nerviosismo, mediante:

a) Expresiones faciales. Para ello se consultó las obras de García y Motos (1990), Günter (1995), Albaladejo (2007), Segura y Arcas (2003) y la web del Centro Aragonés de Tecnologías para la Educación, Portal Aragonés de la Comunicación 
Aumentativa y Alternativa. Se diseñaron ocho cuestiones cerradas de respuesta única mediante imágenes de expresiones faciales, pidiéndole al alumno que relacionara cada imagen (en base a la expresión de los ojos -pregunta 1- o de la boca -pregunta 2-) con la emoción de alegría, enfado, tristeza o sorpresa correspondiente.

b) Lenguaje corporal, manifestaciones fisiológicas. Para ello nos basamos en el artículo de Ruano (2003), la obra de Günter (1995) y la web de la Sociedad Española de ansiedad y estrés. Para esta variable se formularon siete preguntas, de la 3 a la 9, cerradas de respuesta única. En algunas de esas preguntas se le planteaba al alumno una situación emocional y éste debía elegir como respuesta la reacción corporal correspondiente a esa situación y, en otras, las preguntas eran al revés, se describían unos rasgos corporales y fisiológicos en un niño y el alumno debía de reconocer la emoción correcta a esos rasgos. Establecimos tantas preguntas como emociones tratadas en el estudio.

3) Variable de regulación emocional para el miedo, nerviosismo, tristeza y enfado. Para esta variable se hicieron dos preguntas cerradas, la diez y la once, destinadas a la emoción de miedo y nerviosismo y cuatro preguntas abiertas, ya que pensamos que la estrategia de regulación emocional podría ser muy diferente entre un niño y otro y no compartíamos la idea de decidir qué estrategia emocional era mejor que otra. No obstante establecimos esas dos preguntas cerradas a modo de prueba.

En la tabla 1 se sintetiza la estructura del cuestionario, relacionando las preguntas con las variables del estudio.

Tabla 1:

Variables y preguntas del cuestionario

\begin{tabular}{|c|c|c|}
\hline \multicolumn{2}{|l|}{ Variables } & Preguntas \\
\hline \multirow{3}{*}{ Conciencia emocional } & de los demás mediante expresiones faciales & 1,2 \\
\hline & $\begin{array}{l}\text { con uno mismo mediante el lenguaje corporal y } \\
\text { manifestaciones fisiológicas }\end{array}$ & $3,4,5,6$ \\
\hline & $\begin{array}{l}\text { de los demás mediante el lenguaje corporal y } \\
\text { manifestaciones fisiológicas }\end{array}$ & $7,8,9$ \\
\hline Regulación emocional & & 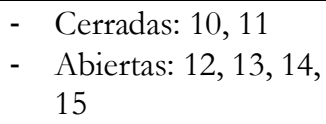 \\
\hline
\end{tabular}

Validación del cuestionario. Se realizó una revisión bibliográfica exhaustiva, extrayéndose los elementos pertinentes derivados de ella; además se utilizó la técnica de jueces (Silva, 1997), primero con un grupo de maestros seleccionados según criterios de años de experiencia en el curso y segundo con un grupo de expertos de EC de la Universidad de Zaragoza. Con ello se generó un primer cuestionario que se aplicó a otra clase semejante $\left(3^{\circ} \mathrm{C}\right)$ a la que teníamos previsto aplicar la unidad didáctica, tras lo cual llegamos al cuestionario final. En esta versión definitiva (ver Anexo I) el cuestionario quedó compuesto por veintiún ítems, diecisiete de ellos fueron diseñados como preguntas cerradas con elección de única respuesta y las últimas cuatro fueron formuladas como preguntas abiertas relacionadas con la variable de regulación emocional.

Aplicación del cuestionario. Este instrumento se aplicó en el horario habitual de clase, antes y después de la unidad didáctica de EC. 
Sistema de valoración. Las preguntas de la 1 a la 11 se valoraron como acierto-error al tener una sola respuesta válida. Las preguntas de la doce a la quince eran abiertas; las respuestas se agruparon en tres categorías a partir de la opinión de los expertos y de la revisión bibliográfica: (1) Ausencia de estrategias (no indica ninguna estrategia de regulación emocional o el contenido de la respuesta es incoherente); (2) Estrategias sin motricidad (indica una estrategia de regulación emocional sin ninguna implicación de aspectos relacionados con la motricidad); y (3) Estrategias con motricidad (indica una estrategia de regulación emocional implicando aspectos relacionados con la motricidad). Análisis de los resultados del cuestionario:

Se calcularon frecuencias y medias de cada ítem del cuestionario y se expresaron en tanto por ciento de aciertos. Para las preguntas abiertas se siguió el mismo tratamiento que exponemos en el diario.

Diario. El diario se iba construyendo en el transcurso de las sesiones. Consistió en una recopilación de comentarios personales escritos que los alumnos vertían sobre situaciones emocionales planteadas por la profesora de EF en la reflexión final de las sesiones. Concluida la sesión, la profesora realizaba dos funciones; una, transcribir los comentarios a un documento Word para luego ser tratados y dos, archivar las fichas de los comentarios cronológicamente en un cuaderno bajo el nombre "En busca de mis emociones", ubicado en el rincón del aula habitual de los niños junto con los otros dos recursos explicados anteriormente.

Tratamiento de la información. Se siguieron los siguientes pasos: (1) Transcripción de las reflexiones; (2) Elaboración de categorías siguiendo unos criterios para su clasificación (ver Tabla 2); (3) Juicio de expertos que revisaron y contrastaron la lista de categorías (4) Categorización y codificación; (5) Codificación de cada reflexión en las categorías establecidas por parte de la profesora de EF; (6) Revisión de dos expertos en EC externos para confirmar la categorización; y (7) Cálculo de frecuencias de cada categoría.

Tabla 2

Relación de las situaciones planteadas con las categorías

\begin{tabular}{|l|l|}
\hline \multicolumn{1}{|c|}{ Situaciones } & \multicolumn{1}{c|}{ Acciones } \\
\hline \multirow{7}{*}{ Validación del cuestionario } & $\begin{array}{l}\text { Se realizó una revisión bibliográfica exhaustiva, } \\
\text { extrayéndose los elementos pertinentes derivados de } \\
\text { ella; además se utilizó la técnica de jueces (Silva, } \\
\text { 1997), primero con un grupo de maestros } \\
\text { seleccionados según criterios de años de experiencia } \\
\text { en el curso y segundo con un grupo de expertos de } \\
\text { EC de la Universidad de Zaragoza. Con ello se } \\
\text { generó un primer cuestionario que se aplicó a otra } \\
\text { clase semejante }\left(3^{\circ} \text { C) a la que teníamos previsto }\right. \\
\text { aplicar la unidad didáctica, tras lo cual llegamos al } \\
\text { cuestionario final. En esta versión definitiva (ver } \\
\text { Anexo I) el cuestionario quedó compuesto por } \\
\text { veintiún ítems, diecisiete de ellos fueron diseñados } \\
\text { como preguntas cerradas con elección de única } \\
\text { respuesta y las últimas cuatro fueron formuladas } \\
\text { como preguntas abiertas relacionadas con la variable } \\
\text { de regulación emocional. }\end{array}$ \\
\hline
\end{tabular}


Tabla 2 (Cont.)

Relación de las situaciones planteadas con las categorías

\begin{tabular}{|c|c|}
\hline Aplicación & $\begin{array}{l}\text { Este instrumento se aplicó en el horario habitual de } \\
\text { clase, antes y después de la unidad didáctica de EC. }\end{array}$ \\
\hline Sistema de valoración & $\begin{array}{l}\text { Las preguntas de la } 1 \text { a la } 11 \text { se valoraron como } \\
\text { acierto-error al tener una sola respuesta válida. Las } \\
\text { preguntas de la doce a la quince eran abiertas; las } \\
\text { respuestas se agruparon en tres categorías a partir de } \\
\text { la opinión de los expertos y de la revisión } \\
\text { bibliográfica: (1) Ausencia de estrategias (no indica } \\
\text { ninguna estrategia de regulación emocional o el } \\
\text { contenido de la respuesta es incoherente); (2) } \\
\text { Estrategias sin motricidad (indica una estrategia de } \\
\text { regulación emocional sin ninguna implicación de } \\
\text { aspectos relacionados con la motricidad); y (3) } \\
\text { Estrategias con motricidad (indica una estrategia de } \\
\text { regulación emocional implicando aspectos } \\
\text { relacionados con la motricidad). }\end{array}$ \\
\hline
\end{tabular}

Para guiar la categorización, se elaboraron diferentes planillas. A modo de ejemplo, incluimos una para la reflexión del día 4 de mayo: "Escribe una situación en la que hayas sentido miedo" (ver Tabla 3).

Tabla 3

Categorías para el análisis de las respuestas a la situación

\begin{tabular}{|l|l|l|l|l|l|}
\hline Criterio & \multicolumn{1}{|c|}{$\begin{array}{c}\text { Nombre } \\
\text { criterio }\end{array}$} & $\begin{array}{c}\text { Nombre } \\
\text { categoría }\end{array}$ & \multicolumn{1}{|c|}{ Descripción } & Código & \% Media \\
\hline \multirow{2}{*}{ CR1 } & \multirow{2}{*}{$\begin{array}{l}\text { Situación de } \\
\text { miedo }\end{array}$} & Real & $\begin{array}{l}\text { Refleja situaciones miedo reales vividas } \\
\text { por los alumnos }\end{array}$ & 1 & 30,4 \\
\cline { 3 - 7 } & Ficticia & $\begin{array}{l}\text { Refleja situaciones de miedo ficticias } \\
\text { descritas por los alumnos }\end{array}$ & 2 & 30,4 \\
\cline { 2 - 6 } & Ns/Nc & Respuestas incoherente o sin contestar & $\mathrm{X}$ & 39,2 \\
\hline
\end{tabular}

\section{Registro en el aula habitual}

Nuestro propósito era realizar un registro diario de las veces que cada alumno utilizaba el "bote vitamínico" (tanto si dejaba como si cogía vitaminas), y la manualidad de "las manos abiertas". Para ello contamos con la colaboración de la maestra tutora, quien iba haciendo el seguimiento de cada alumno mediante una planilla diseñada para ello. Gracias a la colaboración de la tutora pudimos extraer los datos de participación voluntaria de los alumnos ante estas dos estrategias de regulación emocional.

\section{Resultados y comentarios}

\section{Cuestionario de emociones}

Ofreceremos los datos de manera estructurada en función de las variables analizadas en este estudio. 
Conciencia emocional. Las preguntas 1 y 2 relacionaban emociones con expresiones faciales. En el cuestionario inicial dos preguntas, la 1.1 y la 1.3 superan el $100 \%$ de aciertos y el resto de preguntas superaron el 70\%. En el cuestionario final además de estas dos, son 4 preguntas más las que obtienen un $100 \%$ de aciertos y las otras dos están por encima del 75\%. La media de aciertos en el cuestionario inicial fue de $86,1 \%$ y del final fue de $94,1 \%$, por lo tanto ha habido una mejora en la identificación de las emociones en global y, como hemos dicho, en todas las cuestiones o se ha igualado o superado los resultados (ver Tabla 4).

Tabla 4

Conciencia emocional, expresiones faciales

\begin{tabular}{|l|l|l|l|l|l|l|l|l|l|}
\hline & \multicolumn{4}{|l|}{$\begin{array}{l}\text { Pregunta 1 } \\
\text { Identificación a través de los ojos }\end{array}$} & \multicolumn{2}{l|}{$\begin{array}{l}\text { Pregunta } 2 \\
\text { Identificación a través de la boca }\end{array}$} & \\
\hline Apartados & 1.1 & 1.2 & 1.3 & 1.4 & 2.1 & 2.2 & 2.3 & 2.4 & \\
\hline Emoción & Tristeza & Sorpresa & Enfado & Alegría & Tristeza & Sorpresa & Enfado & Alegría & \\
\hline \% inicial & 100 & 71 & 100 & 71 & 88 & 88 & 83 & 88 & 86,1 \\
\hline \% final & 100 & 78 & 100 & 75 & 100 & 100 & 100 & 100 & 94,1 \\
\hline
\end{tabular}

Las preguntas 3, 4, 5 y 6 (conciencia emocional en relación a uno mismo) se referían al reconocimiento del lenguaje corporal y aspectos fisiológicos en situaciones de alegría, miedo, nervios y amor respectivamente. El porcentaje de aciertos en el cuestionario inicial de las tres primeras preguntas rozó el 50\% (46\%, 54\% y 46\% respectivamente) y en el final el 60\% (67\%, 58\% y 46\%). Se mejoraron los aciertos tan sólo en las preguntas 3 y 4. La pregunta 6, aún obteniendo mejoría, obtuvo los valores más bajos (inicial 29\%, final 38\%) de esta variable (ver Tabla 5).

Tabla 5

Conciencia emocional hacia uno mismo (3, 4, 5, 6) y hacia los demás (7, 8 y 9)

\begin{tabular}{|l|l|l|l|l|l|l|l|}
\hline Pregunta & $\mathbf{3}$ & $\mathbf{4}$ & $\mathbf{5}$ & $\mathbf{6}$ & $\mathbf{7}$ & $\mathbf{8}$ & $\mathbf{9}$ \\
\hline Emoción & Alegría & Miedo & Nervios & Amor & Tristeza & Vergüenza & Enfado \\
\hline \% inicial & 46 & 54 & 46 & 29 & 75 & 50 & 71 \\
\hline \% final & 67 & 58 & 46 & 38 & 79 & 58 & 79 \\
\hline
\end{tabular}

Las preguntas 7, 8 y 9 solicitan al niño que reconozca la emoción que siente otra persona; los valores obtenidos en la pregunta 8 están en la línea de otras del cuestionario, pero las preguntas 7 y la 9 (tristeza y enfado respectivamente) destacan porque el porcentaje de aciertos son los más altos con diferencia del cuestionario inicial (75\% y 71\%) y aumentan en el final, siendo de nuevo los más altos $(79 \%)$.

Regulación emocional. Las preguntas cerradas de regulación emocional, es decir la 10 y la 11 , tienen los valores más bajos de todo el cuestionario inicial, destacando especialmente la 10, que tan sólo fue bien respondida por un 4\% de los alumnos; sin embargo está tiene el nivel de mejora más alto de todo el cuestionario, ya que pasa del 4\% al 38\% en el cuestionario final. La pregunta 11 permanece con el mismo porcentaje de aciertos en el final que en el inicial, $29 \%$. 
Tabla 6

Regulación emocional, preguntas cerradas (\%)

\begin{tabular}{|l|l|l|}
\hline Pregunta & $\mathbf{1 0}$ & $\mathbf{1 1}$ \\
\hline Emoción & Miedo & Nervios \\
\hline \% inicial & 4 & 29 \\
\hline \% final & 38 & 29 \\
\hline
\end{tabular}

En las figuras 1y 2 referidas a las preguntas abiertas de regulación emocional, se observa que predominan las estrategias sin motricidad, en especial en el cuestionario inicial (preguntas 12, 13 y 15 con un $65 \%, 83 \%$ y $75 \%$ respectivamente). También se observa un incremento en estrategias con motricidad ante el miedo y nerviosisimo en el cuestionario final. Por último, notamos una disminución en la ausencia de estrategias en las preguntas 12 (de $13 \%$ a $8 \%$ ) y 14 (de $38 \%$ a 22\%), mientras que en la 15 se mantuvo en un $17 \%$ y en la 13 aumentó en el final respecto al inicial (de 4\% a $8 \%)$.

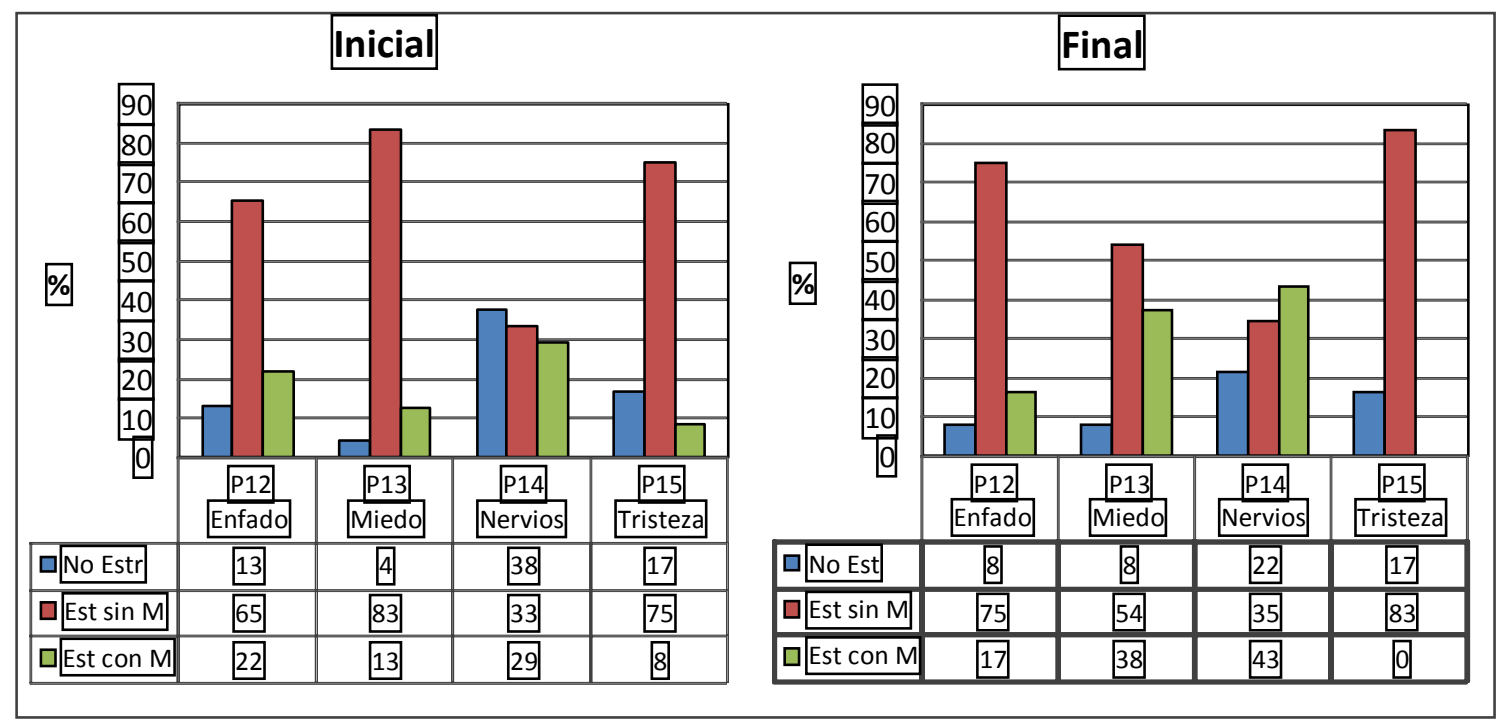

Figuras 1 y 2. \% Respuestas a las preguntas abiertas (\% de cuestionarios inicial y final)

\section{Diario de las emociones} Tabla 7):

Del análisis del diario de las emociones se extrajeron los siguientes resultados (consultar

- Las expresiones de los alumnos al describir situaciones de miedo vividas hicieron referencia tanto a contextos reales como ficticios (real 30,4\%; ficticia 30,4\%).

- Los alumnos al pensar una acción que les provocaba enfado, respondieron con un 45,8\% en acciones en las que intervenía el contacto corporal con el otro, un 29,2\% respondió negativamente pero sin contacto y un $20,8 \%$ indicaron acciones negativas pero sin interacción con los demás.

- Ante esta acción, la mayoría de los alumnos reaccionaban negativamente a través del contacto corporal $(33,3 \%)$ o negativamente pero sin interacción con el otro (29,5\%). Sólo el $8,3 \%$ reaccionaba de forma positiva pero sin contacto y el 12,5\% lo hacía sin interacción. 
Tabla 7

Tipo de acciones y reacciones del alumno (\%)

\begin{tabular}{|l|l|l|l|l|}
\hline & & Contacto corporal & Sin Contacto & Sin Interacción \\
\hline $\begin{array}{l}\text { Acción negativa que produce } \\
\text { enfado }\end{array}$ & 45,8 & 29,2 & 20,8 \\
\hline $\begin{array}{l}\text { Reacciones ante esa acción } \\
\text { negativa }\end{array}$ & Negativas & 33,3 & 12,5 & 29,5 \\
\cline { 2 - 5 } & Positivas & 0 & 8,3 & 12,5 \\
\hline
\end{tabular}

- Los alumnos contextualizaban sus respuestas emocionales en el ámbito de la escuela más que en el de la familia como se puede observar en la Tabla 8.

Tabla 8

Tipo de contexto en el que aparecen emociones (\%)

\begin{tabular}{|l|l|l|l|l|}
\hline \multicolumn{2}{|c|}{} & Nervios & Situación agradable & Situación desagradable \\
\hline \multirow{2}{*}{ Contexto } & Escuela & 66,7 & 83,4 & 54,2 \\
\cline { 2 - 5 } & Familia & 33,3 & 4,2 & 20,5 \\
\hline
\end{tabular}

- Los alumnos sentían más vergüenza cuando interactuaban con el medio u otras personas $(37 \%)$ que en situaciones donde no interactuaban con nadie $(13 \%)$.

- La mayoría de los alumnos pensaban en emociones agradables para transmitir a los demás, adoptando una actitud pasiva (ej. alegría) en vez de activa (ej. dar abrazos) (ver Tabla 9).

Tabla 9

Cuatro sentimientos y reacciones buenas que tengo para transmitir a los demás

\begin{tabular}{|l|l|l|l|l|l|}
\hline \multicolumn{2}{|l|}{} & Media $1^{\text {a }}$ resp & Media 2a resp & Media 3 ${ }^{\mathrm{a}}$ resp & Media $4^{\mathrm{a}}$ resp \\
\hline \multirow{3}{*}{ Actitud } & Actitud pasiva & 62,5 & 50 & 45,8 & 41,7 \\
\cline { 2 - 6 } & Actitud activa & 37,5 & 41,7 & 41,7 & 33,3 \\
\cline { 2 - 6 } & Ns/Nc & 0 & 8,3 & 12,5 & 25 \\
\hline
\end{tabular}

\section{Notas de campo}

De los veinticuatro alumnos, solamente cinco no utilizaron las estrategias de regulación emocional del aula ordinaria ("Bote vitamínico" y "Manos abiertas"). La mayoría de los alumnos las utilizaron una o dos veces. Hubo una minoría que las utilizaron con más frecuencia, dos de ellos 3 veces, y uno 5 veces. 


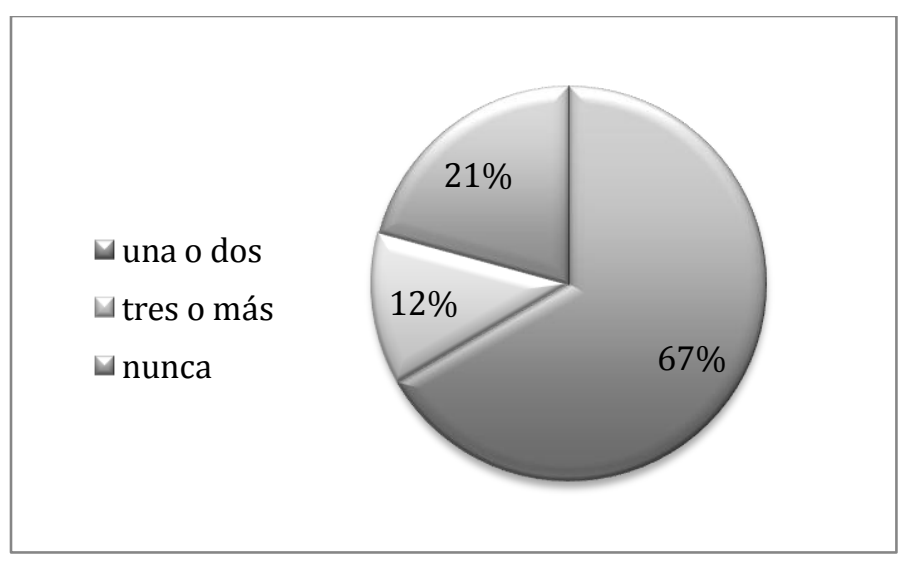

Figura 3. Número de veces que se usaron estrategias en el aula de manera voluntaria

\section{Comentarios sobre los resultados de la experiencia}

Con los resultados del cuestionario, consideramos que el trabajo realizado influyó positivamente en los alumnos, dado que los resultados del cuestionario mejoraron en global y en todos los casos particulares se mantuvieron (pues ya eran del cien por cien) o aumentaron, si bien podrían aplicarse pruebas de contraste en el futuro para confirmarlo.

Algunas cuestiones que nos llamaron la atención fueron que los porcentajes más altos de aciertos del cuestionario hacían alusión al reconocimiento de la emoción de tristeza, enfado, alegría y sorpresa mediante expresión de ojos y boca tanto en el inicial como en el final. No es de extrañar este dato si aludimos a la obra de Abarca (2003), en la que interpretando a Berger (1997) indica que entre los cuatro y siete meses los bebés ya son capaces de asociar las expresiones faciales a su significado emocional.

Por otro lado, las cuestiones con menores porcentajes de aciertos en el cuestionario final correspondían a la identificación de las emociones de amor mediante las manifestaciones fisiológicas y el lenguaje corporal. La emoción del amor algunos autores como Izard (1991), Ekman (1982), Plutchik (1980), García y Motos (1960), expuestos en la obra de Bisquerra (2000), no la clasifican como emoción básica. Esto nos lleva a pensar que quizá no exista una clara diferenciación de esta emoción respecto a otras por parte de los alumnos.

Por otro lado hay que recalcar que se produjo una gran evolución en la regulación de las emociones de miedo y nerviosismo comparando los datos del cuestionario inicial y final, pasando de un $4 \%$ a un $38 \%$ en las preguntas cerradas y, en las preguntas abiertas, de un $13 \%$ a un $38 \%$ en cuanto a utilización de estrategias motrices relacionadas con el miedo y de un $29 \%$ a un $43 \%$ en el caso del nerviosismo. Este aumento pudo ser debido al impacto de las actividades destinadas a regular estas emociones. Sin embargo, los alumnos al final no pensaron en utilizar estrategias de regulación emocional relacionadas con la motricidad para el enfado y la tristeza. En el caso del enfado, este dato nos llevó a pensar que las actividades orientadas para tal fin no calaron lo suficiente en el alumnado. Por otra parte, la emoción de la tristeza no se tuvo en cuenta en la unidad didáctica para trabajarla desde el punto de vista motriz, por lo que parece natural que se produjese esa involución.

De las reflexiones verbales recogidas, se observó que los alumnos siempre orientaban sus respuestas emocionales en el ámbito de la escuela más que en el de la familia. Creemos que la escuela adopta el papel protagonista, pasando a ser el centro de la vida extra familiar del niño. Las relaciones entre iguales aumentan, siendo la escuela el ámbito donde más proliferan este tipo de relaciones. 
Asimismo, creemos que al llevar a cabo la intervención en el ámbito educativo, los alumnos de manera automática daban sus respuestas en este contexto.

En cuanto a cómo sentían las emociones, los alumnos dijeron sentir más vergüenza cuando interactuaban con el medio u otras personas que en situaciones donde no interactuaban con nadie, lo cual está en la línea de Romero Martín (2015), quien considera que dos de los factores clave en la evaluación del grado de vergüenza son la interacción con otras personas o con el medio. También se observó que los alumnos manifestaban emociones positivas hacia los demás, pero adoptaban una actitud pasiva más que activa. Este dato está en la línea de Romero Martín (2015), quien expone que tomar una actitud activa hacia los demás es un paso importante hacia la desinhibición.

Por último, consideramos muy relevante que la mayoría de los alumnos hubieran utilizado las estrategias de regulación emocional propuestas en el aula, ya que requerían de voluntariedad y predisposición del alumno.

\section{Conclusiones}

Una vez realizada la UD y analizados los resultados obtenidos de los instrumentos, pudimos comprobar que hubo cambios relevantes en las competencias de conciencia y regulación emocional. Los alumnos tuvieron mejores resultados en el cuestionario final que en el inicial. Creemos que esta evolución pudo verse influenciada por la unidad didáctica de EC.

Los alumnos evolucionaron en la utilización de estrategias de regulación emocional relacionadas con la motricidad para la emoción del miedo y el nerviosismo tras haber experimentado nuestra propuesta de enseñanza.

Una destacada mayoría de los alumnos utilizaron las estrategias de regulación emocional situadas en el aula ordinaria, en el rincón del aula, al menos una vez durante el periodo que duró la enseñanza.

Se averiguaron aspectos destacables sobre la vivencia emocional de los alumnos que vivenciaron el programa de EC, a través del diario: 1. Los alumnos al reflexionar sobre sus estados emocionales se inclinaron por situaciones donde existía una interacción con el otro. 2. La mayoría de los alumnos orientaron sus respuestas emocionales en el ámbito de la escuela. 3. Casi el total de los alumnos describieron como situaciones de enfado aquéllas en las que había contacto corporal. Asimismo indicaron que reaccionan de manera negativa ante una situación de enfado y que su reacción produce una repercusión negativa en el otro. 4. Los alumnos sienten más vergüenza cuando interactúan con el medio u otras personas que en situaciones donde no interactúan con nadie. 5. Prácticamente el total de los alumnos cuando piensan en emociones agradables para transmitir a los demás, lo hacen adoptando una actitud pasiva (ej. alegría) en vez de activa (ej. dar abrazos).

Una vez concluida la experiencia, vemos que una de las propuestas con alto valor didáctico es hacer un informe de cada alumno con sus características emocionales, partiendo de los datos obtenidos de los instrumentos. Pensamos que puede ser una fuente de información interesante para los agentes de la comunidad educativa. 


\section{Referencias}

Abarca, M. (2003). La educación emocional en la Educación Primaria: Currículo y Práctica. Tesis doctoral. Barcelona: Universidad de Barcelona.

Abarca, M., Barco, L., y Sala, J. (2002). La educación emocional en la práctica educativa de primaria. Bordón. Revista Pedagógica, 54(4), 505-518.

Albadalejo, M. (2007). La comunicación más allá de las palabras: qué comunicamos cuando creemos que no comunicamos. Barcelona: Graó.

Álvarez, M. (2001). Diseño y evaluación de programas de educación emocional. Barcelona: CissPraxis.

Bisquerra, R. (2000). Educación emocional y bienestar. Barcelona: Praxis, D.L.

Bisquerra, R. (2003). Educación emocional y competencias básicas para la vida. Revista de Investigación Educativa, 21(1), 7-43.

Caballero, A. (2004). Cómo enfocar la educación emocional dentro del aula desde la perspectiva de género. En AA.VV., Actas de la Conferencia Internacional Orientación, Inclusión social y Desarrollo de la Carrera (pp. 546-550). La Coruña: Universidad de Coruña.

Castañer, M. y Camerino, O. (2004). Las emociones no escapan a la práctica motriz. La expresión corporal, un procedimiento ideal para trabajarlas. En A. Fraile y J. L. Hernández, Didáctica de la Educación Física: una perspectiva crítica y transversal (pp. 217-236). Madrid: Biblioteca Nueva, D.L.

Castañer, M. y Grasso, A. (2006). La inteligencia corporal en la escuela: análisis y propuestas. Barcelona: Graó.

Centro Aragonés de Tecnologías para la Educación. Portal Aragonés de la Comunicación Aumentativa $\quad$ A $\quad$ Alternativar $\quad$ Rescatado de http://www.catedu.es/arasaac/pictogramas_color.php?Submit=Buscar\&busqueda=basico\& $\mathrm{p}=147 \&$ tipo_palabra $=99 \&$ TXTlocate $=1 \&$ letra $=$ triste $\&$ filtrado $=1 \&$ orden $=$ desc

Extremera, N. y Fernández-Berrocal, P. (2003). La inteligencia emocional: métodos de evaluación en el aula. Revista Iberoamericana de Educación, 30, 1-12. Rescatado de http://www.rieoei.org/psi_edu4.htm

García, L. y Motos, T. (1990). Expresión Corporal. Madrid: Alhambra, D.L.

Gelpi, P., Mateu, M., Romero-Martin, M. R., Rovira, G. y Lavega, P. (2014). La educacion emocional a través de las prácticas motrices de expresión. Perspectiva de género. Educatio Siglo XXI, 32(2), 49-70.

Goleman, G. (1996). Inteligencia emocional. Barcelona: Kairós.

Günter, R. (1995). El lenguaje corporal: lo que decimos a través de nuestras actitudes, gestos y posturas. Madrid: EDAF, D.L.

Lavega, P., Filella, G., Lagardera, F., Mateu, M., y Ochoa, J. (2013). Juegos motores y emociones. Cultura y educación, 25(3), 347-360.

Renom, A. (2003). Educación emocional: programa para Educación Primaria (6-12 años). Barcelona: CISSPRAXIS.

Romero Martín, M. R. (2008). ¿Quo Vadis Expresión Corporal? En G. Sánchez, J. Coterón, J. Gil y Sánchez, A., El movimiento expresivo. II Congreso Internacional de Expresión Corporal y Educación (pp. 105-119). Salamanca: Amarú.

Romero-Martín, M. R. (2015). Expresión Corporal en Educación Física. Zaragoza: Universidad de Zaragoza. 
Ruano Arriagada, K. (2003). ¿Cómo expresamos las emociones? En G. Sánchez y otros, Expresión, creatividad y movimiento. I Congreso internacional de Expresión Corporal (pp. 171-185). Salamanca: Amarú, D.L.

Ruano, T. (2004). Influencia de la Expresión Corporal sobre las emociones. Tesis doctoral. Madrid: Universidad Politécnica de Madrid.

Santiago, P. (1985). De la expresión corporal a la comunicación interpersonal. Madrid: Narcea.

Segura, M. y Arcas, M. (2003). Educar las emociones y los sentimientos: introducción al complejo mundo de los sentimientos. Madrid: Narcea.

Sierra Zamorano, M.A. (2001). La Expresión Corporal desde la perspectiva del alumnado de Educación Física. Tesis Doctoral. Madrid: Universidad Nacional de Educación a Distancia.

Silva Ayçaguer, L.C. (1997). Cultura estadística e investigación científica en el ámbito de la investigación en CC de la Salud: una mirada crítica. Madrid: Díaz de Santos.

Sociedad Española para el Estudio de la ansiedad y el Estrés. Rescatado de http://www.ucm.es/info/seas/ta/index.htm

Torrents, C. y Mateu, M. (2015). Emocionar y emocionarse en movimiento. Tándem Didáctica de la Educación Física, 47, 26-33. 


\section{Anexos}

\section{YO PENSO Y SENTO}

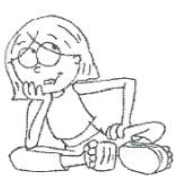

Ahora realizaremos una actividad titulada "Yo pienoo y siento...". La inica regla que nos pide esta actividad es que pensemos sintamos y seamos sinceros. Es necesario que respondamos a todas las preguntas, pero no as preocrupéis, no hay una respuesta luena o maka.

\section{Simplemente hay que pensar, sentir y SER MUY SINCEROS.}

Antes de comenzar con las preguntas rellenaremos muestra ficha personal. Si queréio podéis dibrijar unestra fotografia en el recuadro de la ficha.

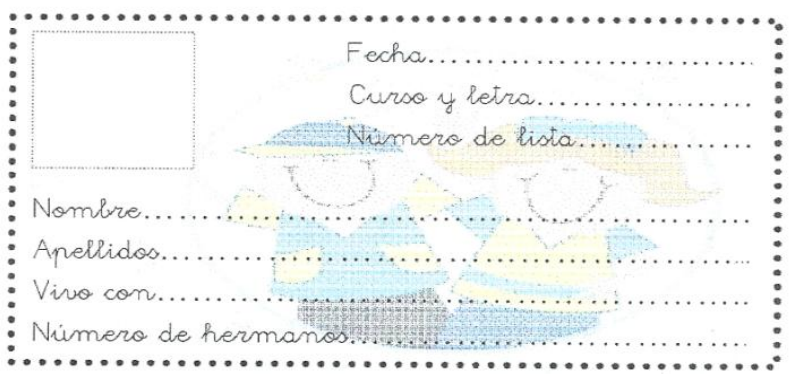

1. Fijate atentamente en la emoción que quieren expresar las caras de estos niñol as. Rodea con un circulo los ojos que correoponden con cada cara.
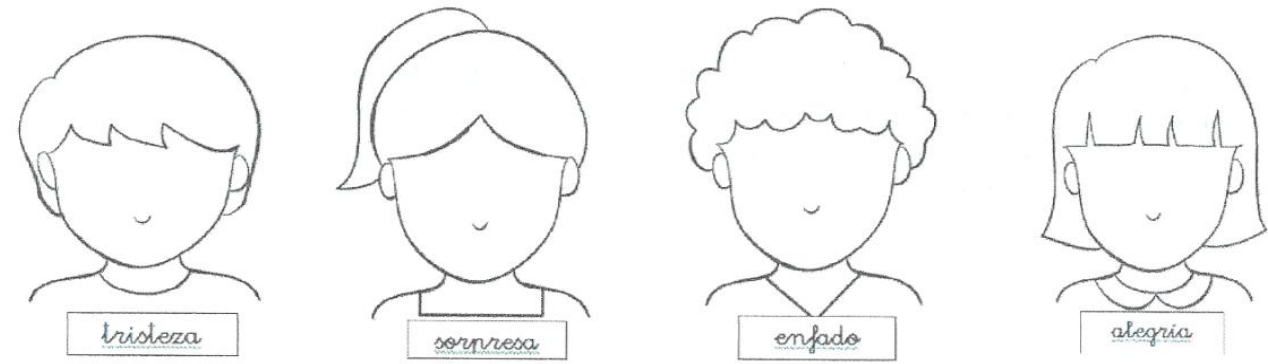
1. ¿ ฉे
2. ๑) ๑
3. 3. 5
4. 1 क ते
5. No lo sé

1. ڤ ఏ

2. बे ब

3. 3 -

4. ब बे

5. No lo sé

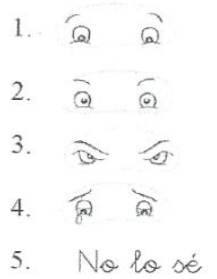

1. இ बे

2. बे อ

3. 35

4. ब खे

5. No lo sé 
2. Fijate atentamente en la emoción que quieren expresar las caras de estos niñol as. Rodea con un circulo la boca que corresponde con cada cara.

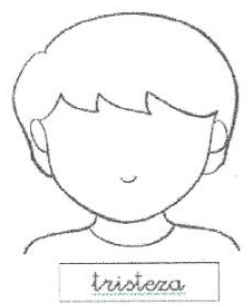

1.

2. 2

3.

4. ح

5. No lo sé

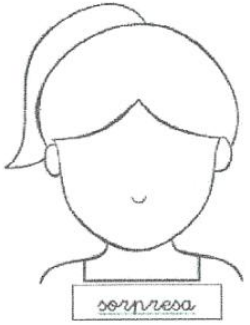

1.

2. 0

3. IIIII

4. $\frown$

5. No lo sé

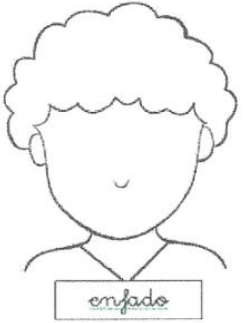

1.

2. 2

3.

4. ᄀ

5. No lo sé

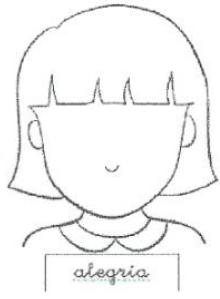

1. $\square$

2. 2

3.

4. ح

5. No lo sé

3. Estás escuchando la canción que más te gusta, y empiezas a bailar, correr, dar saltitos, sintiendo alegria. ¿Cómo te puedes expresar al sentir alegria?

1. Con una sonrisa en la cara y el cuerpo tenso.

2. Con las puntas de lo pies hacia dentro y una sonrisa en la cara.

3. Con una sonvisa en la cara y el cuerpo relajado.

4. Con los brazos cruzados y una sonrisa en la cara

5. No lo sé

4. Imagina que te quedas encerrado en el gimnasio de educación fisica con la huz apagada, y empiezas a tener miedo. ¿Cómo reaccionas al tener miedo?

1. Se me relaja el cuerno. y el corazón me late con rapidez.

2. Se me acelera el corarión y coloco las puntas de los pies hacia dentro.

3. Cruzando los brazos y juntando las cejas.

4. Se me paraliza el cuerpo y el corazión me late con rapidez

5. Nolo sé.

5. Has estado preparando durante una semana un baile para educación fisica, hoy es el día que lo presentas, y estás muy nervioso. ¿Qué reacciones crees que puedes tener?

1. Quedarme totaimente quieto, y sudar más de lo normal.

2. Mover rápidamente los pies y sudar más de lo normar.

3. Sudar más de lo normal y colocar los pies hacia a afuera.

4. Cruzar los brazos y juntar las cejas.

5. No lo sé. 
6. Estas realizando una actividad en educación fisica en la que te sientes muy arropado porque todos the compañeros te dan un abrazo. ¿Qué emoción estás sintiendo?
1. Alegria
2. Vergüenza
3. Sorpresa
4. Amor
5. Ninguna
6. No lo sé
7. Otras..............

7. En clase de edrucación fisica, observas a und a companierol a que parece pensativo, habla menos de lo normal y con woz bajita. Tiene la caberza inclinada, mira al suelo y su sonrisa está al revés. ¿Qué crees que esta sintiendo?

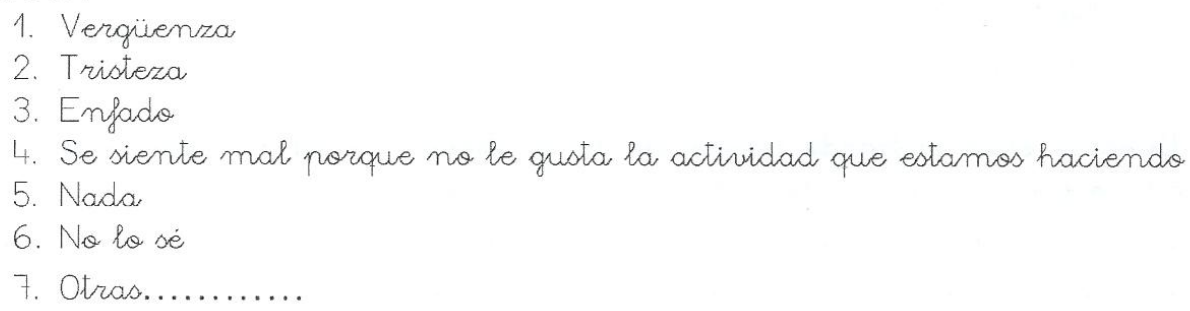

8. Estas realizando una actividad por parejas que consiste en cogerse de las manos y desplazarse por la sala al ritmo de la musica, y observas en the compañerol a que continuamente esta bajando la mirada, evitando mirarte. ¿Qué crees que siente?

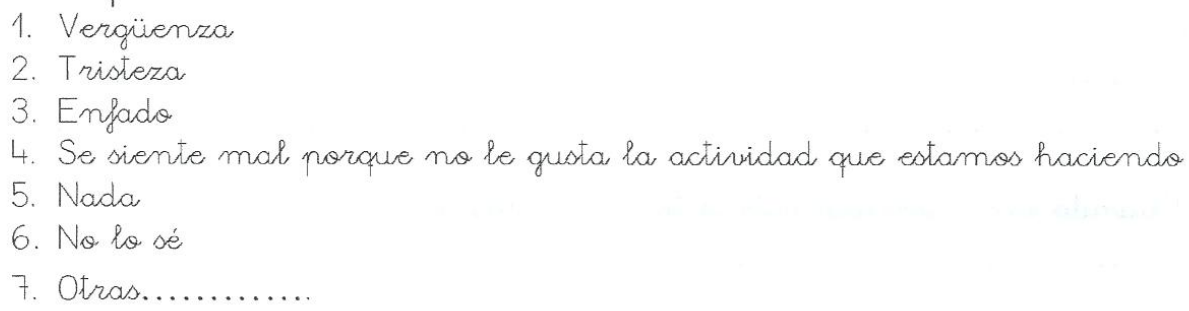

9. Tienes que representar una obra de teatro con un grupo de cinco compañeros. Mientras estáis ensayando, hay un compañero que cruza los brazos y contrae las cejas. ¿Qué crees que siente?

1. Vergïenza

2. Tristerza

3. Enfado

4. Se siente mal porque no le gusta la actividad que estamos haciendo

5. Nada

6. No lo sé

7. Otras............ 
10. ¿Qué harias si te quedas encerrado en el cuarto del material y empiezas a tener miedo?

1. Me quedaria quicto; sin moverme

2. Respiraria como si quisiera enfriar un tazón calentito

3. Empezaria a gritar fuerte y rápidamente

4. No lo sé

5. Otras.............

11. Estás jugando al "robacolas". y un compañero tuyo te quita una cola haciendo trampas. Tí te enfadas y te pones muy nervioso. ¿Qué harias en esa situación?

1. Le quitaria la cola igual que me la ha quitado a mi

2. Contar hasta diez para relajarme

3. Me saldria de la actividad

4. No lo sé

5. Otras...............

12. Cuando estás enfadado Lcómo te tranquilizas? (Ejemplo. Mario cuando esta enfadado piensa en un chiste para tranquilizaroe)

13. Cuando tienes miedo. Lcómo te tranquilizas?

14. Cuando estás nervioso, icómo te tranquilizas?

15. Cuando estás triste Lcómo haces para alegrarte?

Muchas gracias por the esfuerso e interés 


\section{Sobre la autora}

Autor: Laura Luesia Lahoz. DNI 29126275H

Institución: Universidad de Zaragoza. Colegio el Salvador de Zaragoza

E-mail: lluesia@jesuitaszaragoza.es

Información biográfica:

Titulaciones: Diploma de Estudios Avanzados. Programa de Doctorado de Motricidad Humana: Actividades Físicas y Deportivas. Suficiencia investigadora en el Área de Conocimiento de Didáctica de la Expresión Corporal. Universidad de Zaragoza. 2011. Licenciada en Ciencias de la Actividad Física y el Deporte. Instituto Nacional de Educación Física de Cataluña (INEFC) de Lérida. 2006. Universidad Lérida. Maestra especialidad Educación Física. Universidad Zaragoza. 2004. Técnico de Tercer Nivel de la Especialidad de Gimnasia Rítmica. Real Federación Española de Gimnasia. 2008 Experiencia docente: Profesora Asociada en la Facultad de Educación de Zaragoza. Área de Didáctica de la Expresión Corporal. Universidad de Zaragoza. (2007-2009). Maestra de Educación Física en Educación Primaria. Colegio el Salvador de Zaragoza desde 2008 hasta la actualidad. Profesora de Educación Física en Educación Secundaria Obligatoria. Colegio Virgen Blanca. Zaragoza. (2011-2012).

ORCID: no disponible

Autor: María-Rosario Romero-Martín DNI 18418263R

Institución: Universidad de Zaragoza

E-mail: rromero@unizar.es

Información biográfica:

Titulaciones: Doctora en CC de la Actividad Física y el Deporte (Universidad de Zaragoza, 2000). Licenciada en Educación Física (INEF de Barcelona, 1985). Técnica en Expresión (Ayuntamiento de Barcelona).

Puestos docentes: Profesora de Enseñanza Secundaria (Educación Física) desde 1986, actualmente en excedencia. Profesora Titular de Escuelas Universitarias de 1991 a 2003. Profesora Titular de Universidad desde 2003 hasta la actualidad (Área de Didáctica de la Expresión Corporal).

Investigación: 1 sexenio del CNEAI. Coordinadora regional y miembro del equipo investigador de la Red Nacional de Evaluación Formativa y compartida en la educación superior. Miembro de la asociación AFYEC de profesionales de la Expresión Corporal.

Producción científica: Participación en una veintena de proyectos de investigación internacionales, nacionales y regionales. Más de 30 publicaciones científicas en revistas de docencia e investigación en actividad física, participación en congresos con más de 60 comunicaciones, ponencias invitadas y mesas redondas y en más de cincuenta actividades de formación y talleres en centros de profesores, congresos, reuniones científicas y profesionales.

Líneas de investigación: Expresión Corporal, ritmo y danza. Evaluación formativa y compartida. Motricidad Humana. Educación Física. Didáctica de la Actividad física.

ORCID: 0000-0002-5019-4574 


\section{Investigación en la Escuela}

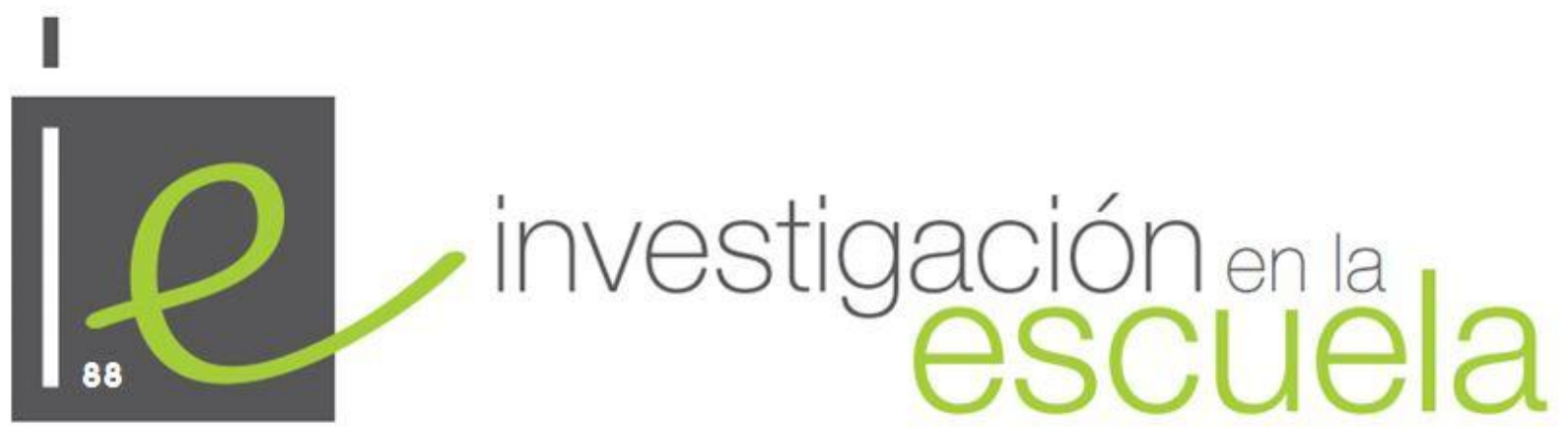

Revista académica evaluada por pares y de acceso abierto

Número 89

15 de noviembre de 2016

ISSN 2443-9991

(c)

SOMERIIGHISRESERVED LOs/as lectores/as pueden copiar, mostrar, y distribuir este artículo, siempre y cuando se de crédito y atribución al autor/es y a Investigación en la Escuela, se distribuya con propósitos no-comerciales, no se altere o transforme el trabajo original. Más detalles de la licencia de Creative Commons se encuentran en http://creativecommons.org/licenses/by-nc-sa/3.0 Cualquier otro uso debe ser aprobado en conjunto por el autor/es, o Investigación en la Escuela.

Contribuya con comentarios y sugerencias en la web de la revista. Por errores y sugerencias contacte a investigacionescuela@,ddcc.uhu.es 


\title{
Investigación en la escuela
}

Consejo de dirección: Ana Rivero García (Universidad de Sevilla), Nicolás de Alba Fernández (Universidad de Sevilla), Pedro Cañal de León (Universidad de Sevilla), Francisco F. García Pérez (Universidad de Sevilla), Gabriel Travé González, (Universidad de Huelva), Francisco F. Pozuelos Estrada (Universidad de Huelva)

\author{
Dirección: Ana Rivero García y Nicolás de Alba Fernández \\ Técnico de edición: Francisco Javier López Sánchez
}

\section{Consejo editorial}

José Félix Angulo Rasco. Universidad de Cádiz Rosa Ma Ávila Ruiz. Universidad de Sevilla Pilar Azcárate Goded. Universidad de Cádiz Juan Bautista Martínez Rodríguez. Universidad de Granada

Nieves Blanco García. Universidad de Málaga Fernando Barragán Medero. Universidad de La Laguna José Carrillo Yáñez. Universidad de Huelva José Contreras Domingo. Universidad de Barcelona. Luis C. Contreras González. Universidad de Huelva Ana $\mathbf{M}^{\mathbf{a}}$ Criado García-Legaz. Universidad de Sevilla Rosario Cubero Pérez. Universidad de Sevilla José $\mathbf{M}^{\mathbf{a}}$ Cuenca López. Universidad de Huelva Jesús Estepa Giménez. Universidad de Huelva Rafael Feito Alonso. Universidad Complutense (Madrid)

Francisco José García Gallardo. Universidad de Huelva

Soledad García Gómez. Universidad de Sevilla J. Eduardo García Díaz. Universidad de Sevilla
Fernando Hernández Hernández. Universidad de Barcelona

Salvador Llinares Ciscar. Universidad de Alicante Alfonso Luque Lozano. Universidad de Sevilla Rosa Martín del Pozo. Universidad Complutense (Madrid)

José Martín Toscano. IES Fernando Herrera (Sevilla) Jaume Martínez Bonafé. Universidad de Valencia F. Javier Merchán Iglesias. Universidad de Sevilla Emilia Moreno Sánchez. Universidad de Huelva. Rosario Ortega Ruiz. Universidad de Córdoba Antonio de Pro Bueno. Universidad de Murcia Fco. de Paula Rodríguez Miranda. Universidad de Huelva

Pedro Sáenz-López Buñuel. Universidad de Huelva Antoni Santisteban Fernández. Universidad Autónoma (Barcelona)

Emilio Solís Ramírez. Catedrático de IES.

$\mathbf{M}^{\mathbf{a}}$ Victoria Sánchez García. Universidad de Sevilla. Magdalena Suárez Ortega. Universidad de Sevilla

\section{Consejo asesor}

Manuel Area Moreira. Universidad de La Laguna

Jaume Carbonell. Director Cuadernos de Pedagogía. Barcelona

César Coll. Universidad de Barcelona

Christopher Day. Universidad de Nothingham. U.K.

Juan Delval. Universidad Nacional de Educación a Distancia

John Elliott. Universidad de East Anglia. Norwich. U.K.

José Gimeno Sacritán. Universidad de Valencia

André Giordan. Universidad de Paris VII y Ginebra

Francisco Imbernón. Universidad de Barcelona

Ángel Pérez Gómez. Universidad de Málaga

Rafael Porlán Ariza. Universidad de Sevilla

Francesco Tonucci. Instituto de Pedagogía del C.N.R. Roma

Jurjo Torres Santomé. Universidad de A Coruña 
